\title{
Synthesis of Mesoporous Ceria by Using CTAB as Template
}

\author{
S. E. Borjas-García ${ }^{1}$, A. Medina-Flores ${ }^{2}$, L. Béjar ${ }^{2}$, P. Martínez-Torres ${ }^{1}$, N. Dasgupta-Schubert ${ }^{3}$, J. L. \\ Bernal $^{4}$ \\ ${ }^{1}$ Instituto de Física y Matemáticas, Universidad Michoacana de San Nicolás de Hidalgo, Morelia, \\ Michoacán, México \\ ${ }^{2}$ Instituto de Investigaciones Metalúrgicas, Universidad Michoacana de San Nicolás de Hidalgo, \\ Morelia, Michoacán, México \\ ${ }^{3}$ Instituto de Investigaciones Químico-Biológicas, Universidad Michoacana de San Nicolás de Hidalgo, \\ Morelia, Michoacán, México \\ ${ }^{4}$ Automotive Mechanics Department. Universidad Politécnica de Pachuca. Zempoala, Hidalgo. México
}

A material which contains pores in a range between 2 and $50 \mathrm{~nm}$ is considered as mesoporous material [1]. The first publication about the synthesis of this kind of material by using an organic template was in 1992 by Mobil scientist [2]. In the case of the synthesis method of mesoporous ceria, different organic templates have been used; for example, cetyltrimethylammonium bromide (CTAB) [3] and triblock copolymer non ionic surfactant [4-6]. However, there is not enough research about the synthesis of mesoporous ceria by using CTAB as template. Mesoporous ceria was synthesized using both Sol - Gel method with a hydrothermal soft treatment. The mesopores of ceria was prepared by using cerium chloride heptahydrate (Sigma-Aldrich), Sodium hydroxide (J.T.Baker) and hexadecyltrimethyl ammonium bromide, CTAB (Sigma, purity) as source, alkaline material and template, respectively. In a first step, two solutions were prepared. The first one was obtained by dissolving $1.863 \mathrm{~g} \mathrm{of} \mathrm{CeCl}_{3} * 7 \mathrm{H}_{2} \mathrm{O}$ and 0.911 of CTAB in $20 \mathrm{~g}$ of distilled water. For the second solution, $1.6 \mathrm{~g}$ of $\mathrm{NaOH}$ was dissolved in $10 \mathrm{~g}$ of distilled water. In a second step, the Na-solution was added slowly (drop by drop) to Ce-solution and stirred. The final solution was stirred and heat in a hot stir plate at $90{ }^{\circ} \mathrm{C}$ to get a material with a molar ratio of $\mathrm{CeCl}_{3} * 7 \mathrm{H}_{2} \mathrm{O}: \mathrm{CTAB}: \mathrm{NaOH}: \mathrm{H}_{2} \mathrm{O}$ equal to 1:0.5:8:150. The gel obtained was aged in a polypropylene bottle at $80{ }^{\circ} \mathrm{C}$ for 1 day. After the hydrothermal treatment, the sample was washed with $100 \mathrm{ml}$ of distilled water and centrifuged at $12000 \mathrm{rpm}$. After that, the material was dried at $80{ }^{\circ} \mathrm{C}$ for 1 day. After the synthesis procedure showed above, the calcination of the sample is necessary at $560{ }^{\circ} \mathrm{C}$ for $6 \mathrm{hrs}$ in order to obtain complete formation of cerium oxide and complete elimination of organic template. The surface morphology and the structural characteristics images of the as-synthesized samples were analyzed by using a scanning electron microscopy FEG-SEM JEOL JSM 7600 and a Tecnai F20 microscope with a field emission gun, respectively. Figure 1a shows an SEM image of ceria synthesized. This figure shows particle size in nanometric scale $(<100 \mathrm{~nm})$. Figure $1 \mathrm{~b}$ shows the EDS spectrum taken over material analyzed. Figure 2 shows the presence of mesopores in the material with a pore size $>2 \mathrm{~nm}$. In this case, the results showed that the molar ratio between cerium and CTAB and hydrothermal treatment temperature could be critical parameters in the formation of mesoporous ceria. Also, medium amount of water in the synthesis procedure allow the formation of mesopores in the material. The synthesis procedure of ceria presented in this research work is easy with a low cost.

\section{References}

[1] D.W. Bruce, D. O’Hare and R.I. Walton, "Porous materials", 1st ed. (Wiley, United Kingdom, 2010) p. 1.

[2] C. T. Kresge et al, Nature Volume 359 (1992) p. 710. 
[3] Daniela Terribile et al, Journal of Catalysis Volume 178 (1998) p. 299.

[4] L. Yue and X. M. Zhang, Ceramics International Volume 35 (2009) p. 847.

[5] Y. Ke and S. Y. Lai, Microporous and Mesoporous Materials Volume 198 (2014) p. 256.

[6] D. Gu and F. Schuth, Chem. Soc. Rev. Volume 43 (2014) p. 313.

[7] The authors acknowledge funding from H. Consejo Técnico of Institute of Physics and Mathematics, and Consejo Nacional de Ciencia y Tecnología (CONACyT), México.
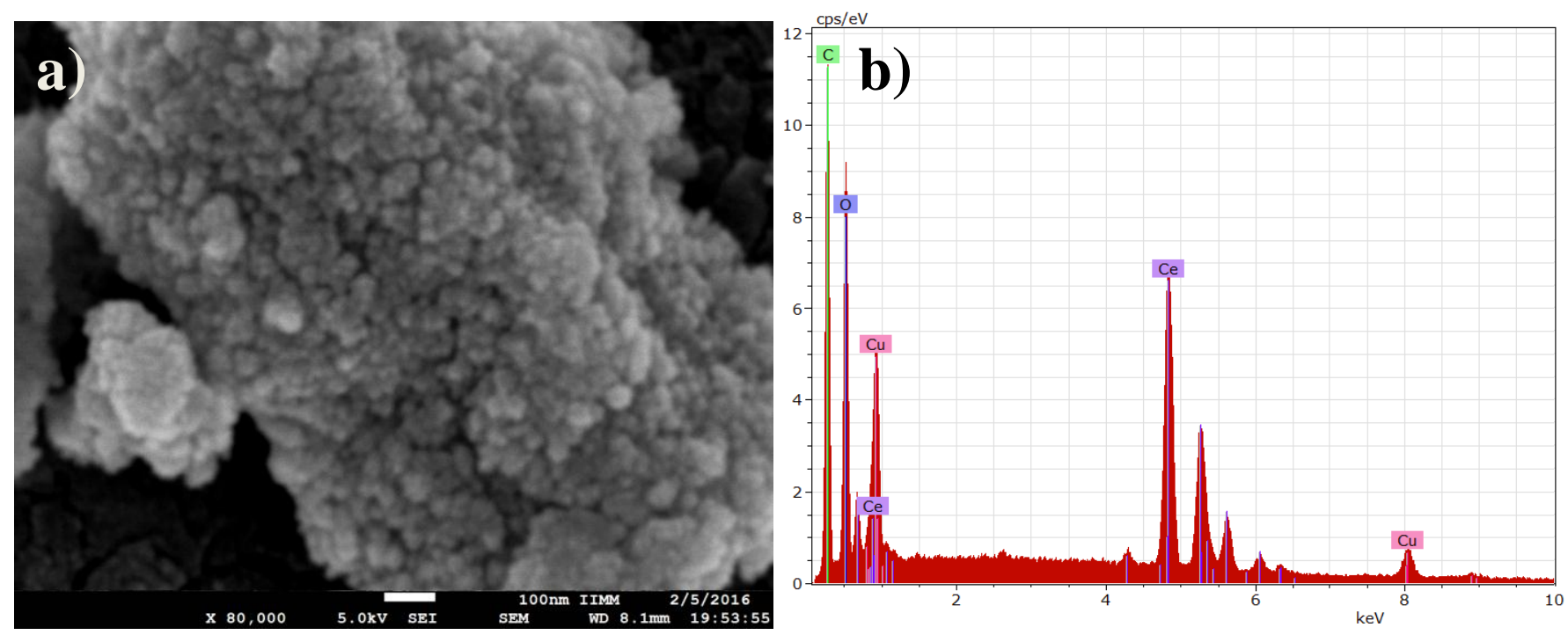

Figure 1. a) SEM image of synthesized material, b) EDS spectrum taken from over one particle.

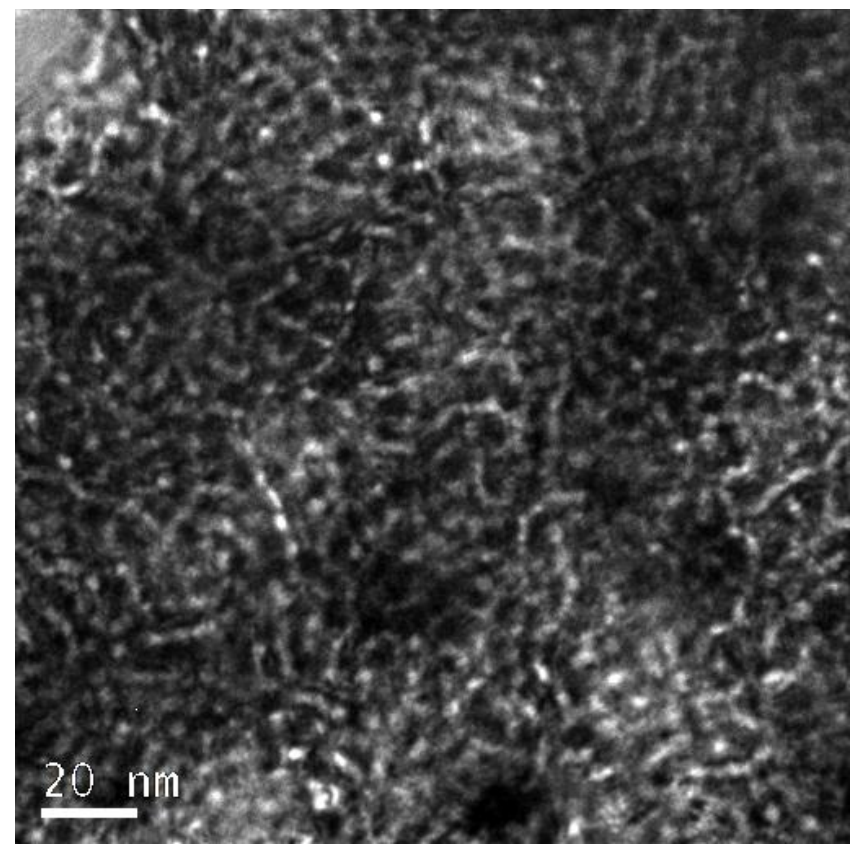

Figure 2. TEM image of mesoporous material after synthesis. 